\title{
Edvard Beneš et la fin de l'Autriche-Hongrie
}

Edvard Beneš und das Ende von Österreich-Ungarn

Edvard Beneš and the End of Austria-Hungary

\section{Antoine Marès}

\section{OpenEdition}

\section{Journals}

Édition électronique

URL : http://journals.openedition.org/austriaca/388

DOI : 10.4000/austriaca.388

ISSN : 2729-0603

\section{Éditeur}

Presses universitaires de Rouen et du Havre

\section{Édition imprimée}

Date de publication : 1 décembre 2018

Pagination : 162-173

ISBN : 979-10-240-1354-1

ISSN : 0396-4590

\section{Référence électronique}

Antoine Marès, « Edvard Beneš et la fin de l'Autriche-Hongrie », Austriaca [En ligne], 87 | 2018, mis en ligne le 01 mars 2020, consulté le 28 janvier 2021. URL : http://journals.openedition.org/austriaca/388 ; DOI : https://doi.org/10.4000/austriaca.388 
Antoine MARÈs

Université Paris Panthéon Sorbonne

\section{Edvard Beneš et la fin de l'Autriche-Hongrie}

Incarnant pour beaucoup ce que l'historien britannique Mark Cornwall a récemment baptisé le czexit ${ }^{1}$, Edvard Beneš requiert deux approches : l'une macro-historique et l'autre micro-historique. Après quelques réflexions liminaires, le parcours de cette personnalité avant 1918, puis les positions qu'il a prises au cours de cette même année permettront de tirer quelques conclusions sur le sujet.

\section{Réflexions préliminaires}

La fin des empires passionne les historiens, depuis L'histoire de la décadence et de la chute de l'Empire romain d'Edward Gibbon ${ }^{2}$ à la fin du $\mathrm{XVIII}^{\mathrm{e}}$ siècle jusqu'à l'essai de Jean-Baptiste Duroselle, Tout empire périra ${ }^{3}$. Dans le public, l'effet de surprise reste dominant : de la même manière que la fascination demeure à propos de l'effacement des dinosaures, comment expliquer que des structures aussi puissantes que les empires disparaissent? C'est l'inquiétude par la taille. Car si les plus gros et les plus forts ont une fin, alors les petits, plus vulnérables, seraient encore plus menacés. Tout particulièrement en Europe médiane, les peurs et le sentiment de faiblesse sont des éléments majeurs de composition du sentiment public, d'autant qu'ils sont cultivés et exacerbés par leur instrumentalisa-

1. Lors de son keynote speech au colloque « Perspectives locales de la sortie de guerre des territoires austro-hongrois » du 14 juin 2018, organisé par Étienne Boisserie (Institut national des langues et civilisations orientales [INALCO], Centre de recherches Europes-Asie [CREE], UMR Sorbonne. Identités, relations internationales et civilisations de l'Europe [SIRICE] et Labex Écrire une histoire nouvelle de l'Europe [EHNE]).

2. Traduit dès 1788 (Paris, Moutard, puis Maradan); disponible sur Gallica dans la traduction de François Guizot (Paris, Ledentu, 1828, 13 vol.).

3. Jean-Baptiste Duroselle, Tout empire périra. Une vision théorique des relations internationales, Paris, Publications de la Sorbonne, 1981. 
tion par les pouvoirs en place. Que ce soit chez les Polonais, les Hongrois ou les Tchèques, la perspective d'une disparition est une angoisse non dissimulée. Et, d'une manière paradoxale, elle peut se nourrir de la réalité, de celle des juifs (ou parfois des Tsiganes) dans la majeure partie de l'Europe médiane pendant la seconde guerre mondiale.

Tout cela donne lieu à des recherches ininterrompues des causes, qui vont de l'idée lancinante de complot ${ }^{4}$ à des raisons sociétales et structurelles en passant par des études plus classiques sur les décideurs, les décisions malheureuses ou les défaites ${ }^{5}$. Il faut cependant noter les différentiels d'interrogations : si l'Empire austro-hongrois reste au cœur des préoccupations avec cette idée longtemps dominante que, s'il avait subsisté, l'Europe n'aurait pas été entraînée dans la deuxième guerre mondiale, la fin de l'Empire ottoman ne suscite pas les mêmes émotions. Elle relève d'une sorte d'évidence, voire d'une justice immanente, contre les oppresseurs et les massacreurs musulmans des chrétiens. La question est donc de savoir si nous pouvons réfléchir sur un principe unique de chute des empires, si nous devons établir une typologie ou si nous devons nous résoudre à considérer que chaque scénario est spécifique et irréductible à d'autres.

Si nous revenons à cette question fondamentale de l'effondrement des empires, soit les explications mettent l'accent sur les décadences et les problèmes internes, soit elles soulignent plutôt les différentiels de puissance et le renversement par des forces supérieures venues de l'extérieur. Le dernier " empire " à s'être effondré au $\mathrm{xx}^{\mathrm{e}}$ siècle a été l'URSS entourée de ses satellites en un temps limité de deux années, entre 1989 et 1991, alors qu'il n'était pas encore cinquantenaire pour sa partie centre-européenne. Son mode d'écroulement soulève d'ailleurs une question inédite puisqu'il s'est produit sans défaite militaire, qui est en général le préalable historique identifié à la chute des empires ${ }^{6}$.

De ce point de vue, la disparition de l'Autriche-Hongrie est un cas classique, de l'Empire romain à l'Empire ottoman en passant par les empires sud-américains, africains ou asiatiques ${ }^{7}$. Un conflit et une défaite

4. Les cibles des thèses complotistes sont, selon les époques et les espaces, les juifs, les francs-maçons, les cosmopolites, les socialistes, le grand capital, le Vatican, les « agents », etc.

5. Corine Defrance (dir.), Vaincus! Histoire de défaites, Paris, Nouveau Monde, 2016.

6. Sur la chute du bloc soviétique, voir l'interprétation exposée par Jacques Lévesque («Essai sur la spécificité des relations entre l'URSS et l'Europe de l'Est de 1945 à 1989 », Relations internationales, $\mathrm{n}^{\circ} 148$, automne 2011, p. 7-16).

7. Antoine Marès et Pierre Boilley (dir.), « Les Empires », Monde(s), n 2, 2012. 
ont débouché sur une disparition, accélérant les tensions internes existantes et provoquant une révolution nationale et une révolution sociale, avec une différenciation selon les espaces nationaux, largement corrélée au sentiment de victoire ou de défaite.

Aujourd'hui, le concept même de « chute d'empire » est mis en question : faut-il parler de chute, de décadence, de déclin ? Ne vaudrait-il pas mieux utiliser des concepts plus fluides comme ceux de transformation, de transition ou d'évolution qui privilégient les continuités sur les ruptures et conduisent à s'interroger sur le temps long ? Et ne faut-il pas prendre en compte dans les analyses l'échelle locale, qui souvent contredit le métadiscours politique tenu à partir du centre ou à partir du seul prisme national ${ }^{8}$ ? L'idée que les empires puissent «mourir " apparait d'autant plus problématique qu'ils peuvent survivre comme mémoire et que, de ce fait, ils peuvent "renaître ", éventuellement sous d'autres formes : sur moins de deux siècles, le cas de la Chine est très intéressant. D’une manière générale, le danger de l'anthropomorphisme guette constamment le discours sur les empires, mais il est inévitable, car la métaphore de la naissance, de la vie et de la mort est irrésistible.

Au-delà de ces métadiscours qui visent souvent à des autolégitimations ultérieures, l'analyse des trajectoires individuelles peut apporter des éclairages pertinents. De ce point de vue, le cas d'Edvard Beneš et de son «pari » tchécoslovaque est particulièrement révélateur de la prévisibilité ou non de la chute de l'Empire austro-hongrois.

\section{Edvard Beneš et les débuts de la guerre}

Il faut tout d'abord régler un débat ancien qui fait de Beneš un nationaliste tchèque antiallemand : la vision historique de Beneš a été de fait déformée par les thèses nazies répandues sur lui - et largement reprises dans la sphère allemande - et par l'expulsion des Allemands dont il est devenu en quelque sorte le paratonnerre ou le bouc émissaire, à travers les prétendus « décrets Beneš », qui résument l'expulsion de l'immense majorité des Allemands des Sudètes installés depuis le Moyen Âge en

8. Voir, sur ce plan, le programme développé par Étienne Boisserie et Catherine Horel au sein de l'INALCO et de l'UMR SIRICE depuis 2017 sur les sorties de guerre austro-hongroises : http://www.inalco.fr/evenement/ecrire-guerre-ecrire-guerre-soldats-civils-empire-austro-hongrois-grande-guerre. 
Bohême ${ }^{9}$. La situation est bien plus complexe et appelle plus que des nuances. Edvard Beneš est né en 1884 dans une famille de paysans pauvres de la Bohême occidentale qui était à l'époque encore fortement binationale ; jeune collégien et lycéen révolté, il se réclamait du marxisme et était alors très proche de la social-démocratie tchèque, sans en être membre. Il se tenait donc à distance des nationalistes tchèques du début du $\mathrm{Xx}^{\mathrm{e}}$ siècle. Il avait même tendance à se battre contre eux, même si le héros hussite Jan Žižka berçait ses rêves d'enfant, et si ce héros hussite a été récupéré par les nationalistes tchèques dans une geste héroïque du passé, figure à la fois structurante de la relation complexe des Tchèques avec le catholicisme et compensatoire d'une histoire où la souveraineté a été confisquée au début du Xvir ${ }^{\mathrm{e}}$ siècle. S'il est anti-habsbourgeois et anti-pangermaniste, c'est plus par idéologie que par germanophobie. Sa proximité avec son professeur Thomas-Garrigue Masaryk ne l'éloigna pas de ses positions sociales-démocrates puisqu'ils avaient en partage des combats communs : l'égalité des femmes, la lutte antialcoolique, la revendication de la journée de huit heures ou la résistance à l'antisémitisme. En revanche, Masaryk avait fait une analyse très critique du marxisme dont se réclamait Beneš quand il avait vingt ans. Quand il décida de poursuivre ses études à Paris à 21 ans (à l'époque, il envisageait d'être enseignant dans le secondaire), il y subsista grâce à ses correspondances au Právo lidu (Le droit du peuple), l'organe de la social-démocratie tchèque. Il n'est pas étonnant dans ces conditions que dans ses travaux universitaires sur la question nationale, il ait été opposé aux thèses nationalistes et à une indépendance tchèque. Dans la thèse qu'il soutint à Dijon le 24 juin 1908, intitulée Le problème autrichien et la question tchèque. Étude sur les nationalités slaves en Autriche, sa conclusion tenait en trois points :

« la question tchèque est le nœud du problème autrichien et sa solution doit nécessairement entraîner la solution de toutes les luttes nationales en Autriche »; « la crise hongroise ne peut être dénouée que par la démocratisation et la décentralisation »;

«l'indépendance complète ne serait pour nous, Tchèques, à l'époque actuelle où se forment exclusivement des Empires immenses, qu' un grand malheur ${ }^{10}$. »

9. Antoine Marès, Edvard Beneš, de la gloire à l'abîme. Un drame entre Hitler et Staline, Paris, Perrin, 2015, et Milan Hauner, " Aux sources de la question allemande chez le jeune Edvard Beneš ", Revue des études slaves, 1998, n 70-4, p. 931-942.

10. Edvard Beneš, Le problème autrichien et la question tchèque. Étude sur les nationalités 
Rappeler cette trajectoire permet de construire l'intelligence des choses. À la veille de la guerre, la position d'Edvard Beneš n'avait pas encore changé. Si, lors de son séjour à Berlin en 1907-1908, il avait vu clairement qu'un affrontement germano-britannique se profilait à l'horizon, il ne croyait pas au scénario qui a suivi et à un enchaînement de Vienne à Berlin, même en 1914, et, bien sûr, il n'envisageait toujours pas la création d'un État tchécoslovaque. En effet, depuis 1848, les Tchèques étaient quasiment unanimes à réclamer une plus grande autonomie dans le cadre d'une fédéralisation de l'Empire habsbourgeois, le fameux " austro-slavisme " de l'historien Franz/František Palacký. Ils vivaient dans la nostalgie des «Articles fondamentaux » de 1871, occasion manquée qui aurait permis d'élargir le Compromis austro-hongrois de 1867 aux Tchèques. Illustrée par le fait que François-Joseph n'a jamais été couronné à Prague et qu'une partie des Tchèques conservait des sentiments mitigés à l'égard de ce "roi sans chapeau ", la déception qui a suivi l'abandon de ce projet de 1871 a dominé la vie politique tchèque jusqu'à la première guerre mondiale. Cependant, seul le Parti de progrès du droit d'État, formation tout à fait marginale, évoquait à la veille de la guerre un conflit général d'où pourrait ressurgir l'ancien royaume de Bohême : cette thèse dite "catastrophiste ", qui mettait l'accent sur une relation étroite entre effort militaire tchèque et recouvrement de l'indépendance, n'était partagée que par quelques individus.

C'est bien à la fin de l'été et au début de l'automne 1914 que la rupture s'est produite, avec une relation étroite entre l'analyse de Thomas-Guarrigue Masaryk et la position d'Edvard Beneš. Après ses contacts avec un certain nombre d'hommes politiques de la Monarchie, allemands ou slaves du Sud, et d'amis de l'Entente (à Rome et à Londres), Masaryk était arrivé à la conviction que les liens entre Vienne et Berlin ne pouvaient plus être rompus. Il en résultait à ses yeux que l'Autriche-Hongrie, dont la légitimité reposait sur son rôle d'équilibre entre Berlin et Moscou, n'avait plus de raison d'être. À partir de ce procès en illégitimité, il prit le parti d'une orientation occidentale pour remplacer l'Autriche-Hongrie et favoriser la (re)naissance d'un royaume de Bohême (très influencé par le modèle britannique, c'était plutôt vers une monarchie constitutionnelle qu'il penchait alors). Et Beneš écrivait dans Lumír en 1915 : 
Il ne s'agit pas, dans le conflit actuel, d'une lutte entre culture occidentale et culture orientale $[\ldots]$. On se bat seulement pour des sphères d'influence et pour des cultures nationales [...]. La guerre, la violence, la révolution sont justifiées et justes; elles sont même beaucoup plus. Elles sont une obligation de chacun si l'on porte véritablement atteinte à la culture spirituelle et matérielle du peuple ${ }^{11}$.

Quand, grâce à un faux passeport grossièrement falsifié et à quelques complicités, Beneš passa clandestinement le $1^{\text {er }}$ septembre 1915 par la ville frontalière de Aš pour rejoindre la Suisse via la Bavière en laissant son épouse Hana à Prague, il tirait donc les conséquences du constat dressé par son maître universitaire Masaryk. Son arrivée à Paris modifia les perspectives de ce groupe d'occidentalistes qui avaient fait le choix de la rupture et leur projet connut des inflexions après la rencontre qui eut lieu à Paris entre Beneš et le Slovaque Milan Rastislav Štefánik du 13 au 15 décembre 1915, d'une part, et l'entrevue de Masaryk avec Aristide Briand, chef du gouvernement et ministre des Affaires étrangères français, en février 1916, d'autre part.

Štefánik conforta l'idée d'une réunion entre Tchèques et Slovaques et insuffla à Beneš son rêve tchéco-slovaque et son énergie visionnaire : on a pu dire que ce duo était l'alliance d'un « artiste » et d'un « laboureur ». En effet, dans le Manifeste du 14 novembre 1915 adopté par les Tchèques de l'étranger, on évoquait encore un "État tchécoslave indépendant ", c'est-à-dire tchèque, et pas un État tchécoslovaque :

Au moment où le noble peuple serbe se ramasse dans un suprême effort pour contenir l'attaque allemande; quand les officiers prussiens et les tortionnaires habsbourgeois, que hante le spectre de la punition prochaine, écrasent sous leurs talons les populations slaves; quand les Dalmates, les Croates, les Slovènes, les Polonais remplissent les cachots et périssent par milliers sur les routes de la déportation et de l'exil ; quand, d'un bout à l'autre de l'Empire, les cadavres des martyrs se balancent au gibet; les Tchèques et les Slovaques, frères de race, de langue et de souffrance, se lèvent à leur tour pour crier aux Habsbourg et à leurs idées leur mépris et leur haine et pour leur déclarer, en face de l'Europe prise à témoin, qu'il ne saurait plus y avoir de commun entre les généreuses nations slaves et les misérables exécuteurs des basses œuvres des Hohenzollern. [...] Ce que nous réclamons désormais c'est un État tchécoslave complètement indépendant. [...] Grâce aux Alliés, la Bohême indépendante et groupant autour

11. "Válka a kultura. Studie z filosofie a sociologie války » (La guerre et la culture. Études de philosophie et de sociologie de la guerre), Lumír, n 43, 1915, p. 219-230 et 262-274, repris en 1922 sous le même titre. 
d'elle tous ses fils, sera, avec la Serbie définitivement délivrée de la menace hongroise, un élément d'équilibre, une garantie de la paix universelle, un ouvrier utile dans le grand atelier de l'humanité ${ }^{12}$.

Quant à la rencontre entre Masaryk et Briand, elle inaugura la phase politique de l'action politique extérieure des exilés. C'est à partir de ce moment que se structura le mouvement de résistance au niveau international et que les exilés misèrent principalement sur Paris, où ils installèrent leur Conseil national au 18 rue Bonaparte, aujourd'hui consulat et Centre culturel tchèque. Beneš a joué un rôle clef dans ce processus, grâce à sa francophonie et francophilie et à son inlassable activité déployée sur les plans de la propagande, de l'organisation politique, de l'armée et finalement de la diplomatie. Il dirigea ainsi la revue $L a$ Nation tchèque, créée par Rudolf Kepl avec l'appui du célèbre historien de la Bohême Ernest Denis, et il se multiplia dans la presse, les salons influents où Štefánik l'introduisit, et les milieux susceptibles d'influencer l'opinion.

Sur le plan de l'organisation, avec son sens de l'autorité, Beneš fit de Paris le centre d'une toile de l'émigration tchéco-slovaque étendue à Londres, Rome, Moscou, Petrograd, Washington, comme secrétaire général du Comité national des Pays tchèques, créé en mars 1916 et transformé quelques mois plus tard en Comité national tchécoslovaque (CNT), sorte de gouvernement des exilés, émigrés, déserteurs ou prisonniers tchèques et slovaques sur les fronts de l'Entente.

Sur le plan militaire, les trois membres du triumvirat Masaryk-BenešŠtefánik et leurs collaborateurs s'efforcèrent de constituer des unités armées en France, en Italie et en Russie : ce qui importait, ce n'était pas leur poids stratégique (globalement, ces troupes ont représenté moins de 100000 hommes), mais leur poids symbolique.

C'est par ces différents canaux que Beneš diffusa un certain nombre d'arguments récurrents jusqu'à la fin de l'année de l'année 1917 à partir de son essai Détruisez l'Autriche-Hongrie. Nous pourrions les résumer à quelques thèmes :

Entourés de tous côtés d'ennemis, assaillis par les troupes prussiennes, maltraités, persécutés et tués, nous avons suivi la voix de notre cœur [...]. Nous n'avons pas reçu de l'Europe des garanties certaines de notre libération et les puissances de l'Entente ne se sont pas prononcées encore en notre faveur. Nous n'avons pas

12. La Nation tchèque, $3^{\mathrm{e}}$ année, $\mathrm{n}^{\circ} 14,15$ novembre 1915, p. 215. 
attendu ces garanties et nous nous sommes, malgré cela, placés à leurs côtés, car le peuple de Jean Hus, de Komensky, de Kollar et de Palacky ne pouvait agir autrement. Nous ne sommes pas venus en France demander en gémissant à l'Europe qu'elle ne nous laisse pas écraser sous le joug du pangermanisme. Ce que nous avons fait, nous l'avons fait parce que c'était notre devoir. Nous sommes venus montrer par nos actes, par notre conduite, par notre passé quelles étaient nos traditions, quelles ont été nos luttes et quelles sont nos ambitions actuelles $^{13}$.

Il martelait un certain nombre d'arguments : " La libération des Tchécoslovaques est la condition indispensable de la paix »; " déclarer dans la réorganisation future de l'Europe centrale la Bohême libre, réunie aux Slovaques du nord de la Hongrie, constituera une garantie de premier ordre pour la paix future de l'Europe »; la " Bohême est un rempart dressé par la géographie pour lutter contre le pangermanisme, c'est sur elle que la France devra s'appuyer ${ }^{14}{ }^{\prime}$.

La démonstration du CNT s'articulait ainsi autour de trois axes : l'unité du mouvement tchèque, même si elle était bien compromise par l'évolution interne de la monarchie ; les manœuvres qualifiées de "trompeuses " des Autrichiens, tandis qu'à Vienne même on ne croyait plus à la victoire; et la mise sur pied d'un État tchécoslovaque, qui était le dernier espoir d'une barrière antigermanique à l'Est, alors que les Polonais semblaient se contenter d'une Pologne reconstituée sous l'égide germano-autrichienne. Encourager les Tchèques, ce serait donc aussi convaincre les Polonais de suivre une autre voie. Le nouvel État tchécoslovaque constituerait « une barrière antigermanique et l'obstacle principal au plan pangermanique ». Le pays, économiquement puissant, formerait avec l'Italie et la Yougoslavie un mur infranchissable. Au nord, les Polonais seraient des alliés militaires. Les restes de l'Autriche-Hongrie seraient donc bloqués par trois groupes : polono-tchèque, russo-roumain et balkanique ${ }^{15}$. Il faudrait cependant pour cela que l'Entente ne fût pas tentée par une fédéralisation utopique de la Monarchie, tant Vienne avait partie liée avec Berlin.

13. Edvard Beneš, Détruisez l'Autriche-Hongrie! Le martyre des Tchéco-Slovaques à travers leur histoire, Paris, Librairie Delagrave, 1916. La carte jointe a été supprimée par la censure dans les éditions française et anglaise (Archives historiques militaires tchèques, VHA, ČSNR, $\left.\mathrm{n}^{\circ} 14\right)$.

14. Antoine Marès, Edvard Beneš, op. cit., p. 95.

15. Archives du ministère de l'Europe et des Affaires étrangères, La Courneuve, série Guerre 1914-1918, nos 153 et 154 (rapport du 28 novembre 1917 à Pierre de Margerie). 


\section{L’année 1918}

Le programme tchèque fit l'objet d'un nouveau mémorandum remis au Quai d'Orsay le 29 mai $1918^{16}$. Outre les provinces historiques (Bohême, Moravie et Silésie) et la Slovaquie, les Tchèques y revendiquaient les régions de Glatz et de Ratibor, soit un ensemble de $140000 \mathrm{~km}^{2}$ peuplé, selon leurs estimations (erronées), de 10 millions de Tchécoslovaques et d'1,5 million d'Allemands, de Polonais et de juifs, un chiffre notoirement sous-estimé, puisque les Allemands seront plus de 3 millions. Ainsi, un bloc polono-tchèque de 40 millions d'habitants, uni politiquement, militairement et économiquement, serait-il constitué, dans lequel la Bohême jouerait un rôle moteur grâce à son industrie et à sa stabilité politique. Cette Tchécoslovaquie pourrait avoir une frontière avec la Roumanie sur la haute Tisza où les Slovaques voisinent avec les Ruthènes et les Roumains. Reliés par le Danube et par une nouvelle voie de chemin de fer, les deux pays auraient des liens économiques importants. Les relations privilégiées de la Bohême et de la Yougoslavie se poursuivraient et la "mer yougoslave " serait aussi tchécoslovaque grâce à la construction d'une voie ferrée allant de Presbourg (Bratislava) à l'Adriatique. Les échanges seraient également importants avec l'Italie et, dans cet édifice, la France jouerait le rôle de tutrice et de grande puissance protectrice. Beneš s'abstint de mentionner le couloir tchécoslovaco-yougoslave évoqué dans sa brochure de 1916, qui aurait séparé l'Autriche de la Hongrie et permis une liaison avec l'allié potentiel au $\operatorname{sud}^{17}$, idée dont quelques Français se faisaient les défenseurs ${ }^{18}$.

À la fin des offensives allemandes du printemps 1918, Edvard Beneš confirma à ses interlocuteurs praguois les progrès réalisés, la «situation extraordinaire » de la question tchèque et, en même temps, il annonçait une possible déroute militaire qui guettait les Alliés face à l'offensive allemande. Ces réactions au jour le jour traduisaient le désarroi face à une situation kaléidoscopique. Il n'excluait donc pas encore un retournement de situation, une victoire des puissances centrales et une défaite de ceux qui espéraient la fin de l'Empire habsbourgeois. Ceci confirme la pertinence des interrogations sur l'inévitabilité de la création de la Tchécoslovaquie et de la disparition de l'Autriche-Hongrie.

16. Ibid., $\mathrm{n}^{\circ} 154$.

17. Détruisez l'Autriche-Hongrie, op. cit., p. 70.

18. Arthur Chervin, De Prague à l'Adriatique, Paris, Berger-Levrault, 1919. 
Une fois l'unité des Tchèques et des Slovaques assurée, une fois leur volonté de combattre auprès de l'Entente démontrée, il était possible de passer à l'action diplomatique : le tournant décisif fut pris de ce point de vue au printemps 1918, quand le président du Conseil français Georges Clemenceau annonça le 20 avril à Beneš qu'il considérait la question tchèque comme résolue. Cette confidence était directement liée à l'incident qui avait opposé le comte Czernin, ministre des Affaires étrangères autrichien, et Clemenceau, à propos des contacts secrets initiés par Charles ${ }^{\text {er }}$ pour explorer les voies d'une possible sortie de guerre. Ici encore, il faut se reporter aux débats parlementaires de l'époque pour comprendre à quel point la vision d'un Clemenceau souhaitant la disparition de l'Autriche-Hongrie depuis le début de la guerre est une légende. Comme il le dit et le fait dire à ce moment, la France n'avait pas de politique autrichienne ${ }^{19}$. Et ce fut bien le cas jusqu'au moins l'hiver 1917-1918.

Le processus de reconnaissance de la future Tchécoslovaquie avait en effet déjà été entamé par le décret constitutif de l'armée tchécoslovaque en France en décembre 1917, négocié pas à pas par Beneš ; il avait vainement tenté de profiter de cette rédaction pour obtenir une reconnaissance implicite du futur État, ce à quoi il n'était pas arrivé immédiatement. Elle ne fut parachevée qu'avec la remise du drapeau aux troupes tchécoslovaques le 30 juin 1918 à Darney, dans les Vosges, par le président de la République française Raymond Poincaré : « Le jour qui se lèvera sur une Alsace-Lorraine libérée, éclairera d'un rayon nouveau toutes les nations martyres. Allez au combat, Messieurs, et vous verrez bientôt les premiers feux de cette aurore ${ }^{20}$. " Beneš représentait le CNT à cette cérémonie, dont l'anniversaire est devenu en 2002 la fête des Forces armées de la République tchèque ${ }^{21}$; la veille, il avait reçu une lettre du ministre des Affaires étrangères français reconnaissant le CNT comme "première assise du gouvernement tchécoslovaque ». La question n'était pourtant pas encore réglée et les mois suivants furent marqués par la crainte constante d'un découplage entre le CNT et les acteurs praguois, dont certains - il est vrai de moins en moins nombreux - étaient toujours enclins au compromis avec les autorités.

19. Archives nationales, C 7491, Chambre des députés. Voir aussi Jean-Baptiste Duroselle, Clemenceau, Paris, Fayard, 1988, p. 812-813.

20. Raymond Poincaré, Messages, discours, allocutions, lettres et télégrammes. Vol. 3 : Août 1919-février 1920, Paris, Bloud et Gay, 1919, p. 245.

21. Grâce notamment à l'action de l'ambassadeur tchèque en France, le francophile Petr Janyška. 
C'est fort de la reconnaissance française de juin 1918 que Beneš arriva le 28 octobre à Genève pour rencontrer une délégation d'hommes politiques tchèques chevronnés, avec Karel Kramáŕ à leur tête : impressionnés par le jeune ministre des Affaires étrangères devenu leur égal, ils décidèrent d'adopter le régime républicain, de nommer Masaryk président de la République et Kramár président du Conseil. Ils ne savaient pas encore que, dans la matinée, d'autres leaders politiques tchèques avaient proclamé l'indépendance à Prague. Beneš informa alors les autorités françaises :

Si, par impossible, l'Entente s'efforçait de sauver l'Autriche et y réussissait, la révolution éclaterait à Prague et, avec elle, sans doute le bolchevisme [...] Le bolchevisme grandit partout en Europe orientale et seuls les Tchèques sont capables, avec l'aide de l'Entente, d'en enrayer les progrès ${ }^{22}$.

Présentant le nouvel État à la fois comme une citadelle industrielle, une barrière contre l'Allemagne et une barrière contre le bolchevisme, il déploya ainsi une rhétorique forte qu'il mit au service de la construction de l'État tchécoslovaque. Car tout restait à faire : la Tchécoslovaquie reconnue par l'Entente n'avait encore ni administration nationale, ni frontières, et les nouvelles forces minoritaires contestaient son existence. Une des premières lettres écrites par Beneš à son épouse au lendemain de la guerre (le 19 novembre) témoigne de vertige et de sidération qui saisit le nouveau ministre des Affaires étrangères devant ce " miracle » tchécoslovaque :

Moi-même, je n'arrive pas à comprendre parfois tout ce qui s'est passé, comment il est possible que toutes ces choses aient convergé entre mes mains et que j'aie ce rôle à jouer. J'ai beaucoup travaillé, beaucoup fait, j'ai connu beaucoup de choses et $\mathrm{j}$ 'ai beaucoup appris, mais tout cela, un tel succès, un tel triomphe, je ne les attendais pas. Il est vrai aussi qu'on a bien travaillé ici et que des miracles ont eu lieu. Je te raconterai tout, comme c'est beau et instructif ${ }^{23}$.

L'essentiel était alors de justifier les revendications du nouvel État auprès des décideurs de la Conférence de la Paix et des experts qui y

22. Antoine Marès, Edvard Beneš, op. cit., p. 113.

23. Listy důvěrné. Vzájemná korespondence Hany a Edvarda Benešových (Lettres confidentielles. Correspondance croisée de Hana et Edvard Beneš), Hana Šetřilová et Jaroslav Čechura (éd.), Prague, Česká expedice-Rio Press, 1996, p. 29. 
travaillaient : le Paris de 1919, c'était la Vienne de 1815. On y dansait, on y déployait ses talents de séduction (et de corruption), on y organisait des concerts du fameux Quatuor tchèque. Beneš s'imposa rapidement par son sérieux et sa modération de ton : il sut écarter les revendications extrêmes (sur l'annexion des Sorabes de Lusace ou sur le couloir de liaison avec la Yougoslavie entre l'Autriche et la Hongrie), invoquer des arguments géostratégiques auxquels ses interlocuteurs étaient sensibles et apparaître comme un homme de paix. Il sut aussi tirer habilement profit des circonstances en misant sur une alliance privilégiée avec la France à qui il décida de confier début décembre 1918 la construction de l'armée tchécoslovaque. Ce fut d'ailleurs la Mission militaire française qui, au printemps 1919, renversa la situation en Slovaquie en faveur de Prague débordée par l'armée hongroise des Conseils. Quand il décida de revenir dans sa patrie après avoir signé les traités de Versailles et de Saint-Germain-en-Laye, l'essentiel était fait, la Tchécoslovaquie était consolidée et son entrée en Tchécoslovaquie et à Prague fut triomphale.

Retournant en Tchécoslovaquie en septembre 1919, cinq ans après son départ, ovationné par les foules de la frontière autrichienne à Prague, Edvard Beneš bénéficiait à 35 ans de l'immense aura de son succès dans la défense des intérêts du nouvel État. À la tête du ministère des Affaires étrangères, il devait désormais construire une politique étrangère en vue d'assurer la sécurité de son pays : une tâche difficile et périlleuse après la disparition de la Russie des relations internationales, le repli sur soi des États-Unis, les dissensions entre la France et la Grande-Bretagne et la présence aux frontières de la Tchécoslovaquie de voisins insatisfaits attendant de prendre leur revanche. Pourtant, son extraordinaire ascension de fils de paysan pauvre de Kožlany avait renforcé sa confiance absolue en son étoile et en son destin : seul le traumatisme des accords de Munich allait entamer cette conviction deux décennies plus tard. Il avait le sentiment d'avoir " enfanté » la Tchécoslovaquie qu'il a toujours considérée comme son " enfant ", et il était persuadé que son approche « scientifique » de la politique étrangère lui assurerait un succès permanent.

\section{Conclusions}

Pour en revenir à la question initiale des causes de l'éclatement de l'Autriche-Hongrie et de la naissance de la Tchécoslovaquie, il faut souligner que la fin de cet Empire n'était pas prévisible au début de la 
guerre, ou du moins qu'il n'y avait aucune fatalité à sa disparition : le loyalisme tchèque était dominant (même si, a contrario au sein de l'émigration tchèque, il s'exerçait en faveur de l'Entente, en Russie, aux ÉtatsUnis ou en France). Et, dans les chancelleries, la tendance était également au maintien de cet État parce que personne n'imaginait par quoi le remplacer. Le sentiment que l'Autriche-Hongrie était une " prison des peuples ", argument de propagande anti-habsbourgeoise développé par les exilés, était d'ailleurs très inégalement partagé parmi les peuples de la Monarchie (par exemple chez les Polonais ou chez les catholiques tchèques, il était quasi inexistant) et à l'extérieur (à cet égard, Cisleithanie et Transleithanie doivent être distinguées).

Dans le cadre des uchronies à la mode - qui mettent en jeu un raisonnement contrefactuel sur des futurs non advenus -, nous pourrions formuler l'hypothèse suivante à propos de l'année 1917 : si, au moment où Charles annonça la réunion du Parlement de Vienne pour la fin mai avant d'amnistier les condamnés politiques pour trahison en été, il avait proposé une fédéralisation générale de l'Autriche-Hongrie, il aurait très vraisemblablement désarmé ceux qui voulaient la disparition de cette dernière. Quand il le fit à la mi-octobre 1918, et seulement pour la Cisleithanie, il était trop tard. La radicalisation nationale et sociale qui a fonctionné en vases communicants en 1917 et 1918 en raison des difficultés liées à la guerre, tant sur le front qu'à l'arrière, a eu pour effet que plus aucun peuple ne voulait vivre ensemble. Cela touchait évidemment les nationalités centrifuges (Tchèques, Serbes, Roumains, puis Polonais et Croates, et dans une moindre mesure les Slovènes) tout comme les Allemands d'Autriche, qui souhaitaient leur rattachement à l'Allemagne, et les Hongrois, qui cherchaient à parachever leur indépendance.

Quant à l'exemple de Beneš, il montre que, quels que soient ses engagements à partir de 1915-1916 en faveur de la mise sur pied d'un État tchécoslovaque dont les contours précis restaient encore flous, il n'exclut pas l'échec jusqu'à une date tardive, même s'il le masqua dans ses Mémoires ${ }^{24}$. Tout était suspendu à la victoire finale de l'Entente, qui ne s'affirma qu'en été 1918. Le retour aux sources permet de déconstruire les discours étatiques auto-légitimateurs de l'après 1918.

24. Edvard Beneš, Souvenirs de guerre et de révolution (1914-1918), Paris, Ernest Leroux, 1928. 